\title{
Correlation of the phenotypic ethambutol susceptibility of Mycobacterium tuberculosis with embB gene mutations in Korea
}

\begin{abstract}
Correspondence
Young Kil Park

ypark7@empal.com
\end{abstract}

Received 26 August 2011

Accepted 8 December 2011

\author{
Young Kil Park, Sung Weon Ryoo, Seung Heon Lee, Hum Nath Jnawali, \\ Chang-Ki Kim, Hee Jin Kim and Sang Jae Kim
}

\author{
Korean Institute of Tuberculosis, 482 Mansuri, Kangwoimyun, Cheongwongun, Chungbuk, \\ Republic of Korea
}

\begin{abstract}
The phenotypic resistance to ethambutol (EMB) in Mycobacterium tuberculosis with embB gene mutations is still unclear. This study was designed to better understand EMB resistance due to embB gene mutation. Sequencing analysis of the embB gene was performed for 124 EMBsusceptible and 93 EMB-resistant M. tuberculosis strains isolated from South Korea. The MIC was determined for EMB-susceptible M. tuberculosis strains with the embB mutation and wildtype on Löwenstein-Jenson (LJ) solid medium in duplicate. Two (2.8\%) of 72 pan-susceptible, two $(9.1 \%)$ of 22 any-drug-resistant but EMB-susceptible, nine (30.0\%) of 30 multidrugresistant (MDR) but EMB-susceptible and 84 (90.3\%) of 93 EMB-resistant M. tuberculosis strains possessed embB mutations at various codons including 306, 319, 354, 360, 399, 405, 406, 459 and 497. Strains with embB mutations at codons 306, 354, 399, 405 and 497 had highly pronounced EMB resistance, while strains with mutations at codons 319 and 406 mutations were moderately resistant and those with an embB459 mutation were EMBsusceptible at the critical concentration $\left(2.0 \mu \mathrm{g} \mathrm{ml}^{-1}\right)$ on $\mathrm{LJ}$ solid medium. However, the mean MIC for strains with embB mutations $\left(1.42 \mu \mathrm{g} \mathrm{ml}^{-1}\right)$ was higher than that for strains without the embB mutation $\left(1.0 \mu \mathrm{g} \mathrm{ml}^{-1}\right)$ in EMB-susceptible $M$. tuberculosis isolates $(P=0.0052)$. Three novel embB mutations at codons 399, 405 and 459 were identified in this study. These results support the hypothesis that embB mutation except for a few specific mutation types may be the main cause of EMB resistance.
\end{abstract}

\section{INTRODUCTION}

Tuberculosis is a major global health problem (WHO, 2009). The emergence of multidrug-resistant (MDR) tuberculosis and, more recently, of extensively drug-resistant tuberculosis, is widely considered to be a serious threat to global tuberculosis control (CDC, 2006; Migliori et al., 2007; Raviglione \& Smith, 2007). The control of tuberculosis remains one of the more elusive goals in medicine. Rapid detection of drug resistance is essential to design appropriate treatment regimens, prevent treatment failure and thus reduce the further spread of drug-resistant isolates.

Ethambutol (EMB) [dextro-2,2' -(ethylenediimino)-di-1butanol] is a narrow-spectrum antimycobacterial agent that is used for the treatment of tuberculosis. EMB is a first-line anti-tuberculosis agent that is especially important when used in drug combinations to prevent the emergence of drug resistance or to treat single drugresistant tuberculosis (WHO, 1997). Further, streptomycin

Abbreviations: $\mathrm{EMB}$, ethambutol; MDR, multidrug-resistant.

A supplementary table is available with the online version of this paper. has been replaced by EMB as a key drug in the intensive phase of tuberculosis chemotherapy as it is less expensive and patient compliance is better with this drug (Rabarijaona et al., 1999). This agent has been proposed to be an arabinose analogue; the specific target is likely to be an arabinosyltransferase, presumably a functionally important site. A two-gene locus (embAB) that encodes arabinosyltransferase has been studied to elucidate a potential mechanism of EMB resistance (Alcaide et al., 1997; Telenti et al., 1997).

Amino acid replacements at position 306 of EmbB have been shown in many studies to be present in EMB-resistant, but not EMB-susceptible, organisms (Alcaide et al., 1997; Ramaswamy \& Musser, 1998; Sreevatsan et al., 1997). However, Mokrousov et al. (2002) detected mutations of embB306 in both EMB-susceptible and EMB-resistant strains. EMB is an important antimycobacterial drug as it enhances the effect of other companion drugs including aminoglycosides, rifamycins and quinolones. Moreover, this drug has also been shown to significantly decrease the levels of bacteraemia in patients with AIDS (Kemper et al., 1994). 
Consequently, mutations in codon 306 have been suggested as molecular markers for the rapid detection of EMB resistance (Lee et al., 2004; Plinke et al., 2006; Van Rie et al., 2001). Furthermore, two recent studies demonstrated a clear causative relationship between embB306 point mutations and in vitro EMB resistance by allelic-exchange experiments (Safi et al., 2008; Starks et al., 2009). Identification of additional mutations occurring in EMB-resistant organisms will be useful in further understanding the mechanisms of resistance to this primary anti-tuberculosis agent. In our study, we tried to understand EMB resistance in detail by studying its relationship with mutations in the $e m b B$ gene.

\section{METHODS}

Strains. The strains were randomly selected from among $M$. tuberculosis isolates from pulmonary tuberculosis patients registered in public health centres of South Korea from 2005 to 2007 that were stored at the Korean Mycobacterial Resource Center (KMRC). We included 72 pan-susceptible, 22 EMB-susceptible with any drug resistance but non-MDR, 30 EMB-susceptible and MDR and 93 EMB-resistant with or without other drug resistances (Supplementary Table S1 in JMM Online). All strains had different IS6110 RFLP types, as performed by a standardized procedure described previously (Park et al., 2000).

Drug susceptibility testing. Phenotypic drug susceptibility testing was performed against isoniazid $\left(0.2 \mu \mathrm{g} \mathrm{ml}{ }^{-1}\right)$, rifampicin $(40 \mu \mathrm{g}$ $\left.\mathrm{ml}^{-1}\right)$, streptomycin $\left(10 \mu \mathrm{g} \mathrm{ml}^{-1}\right)$ and $\operatorname{EMB}\left(2 \mu \mathrm{g} \mathrm{ml}^{-1}\right)$ in LJ medium using the absolute concentration method (Canetti et al., 1969). The MICs of EMB were compared between pan-susceptible strains and EMB-susceptible but other-drug-resistant $e m b B$ mutant strains.

Selected isolates were inoculated onto $\mathrm{LJ}$ medium containing various concentrations of $\operatorname{EMB}\left(0-32 \mu \mathrm{g} \mathrm{ml}^{-1}\right)$. These strains were retested twice to obtain a mean EMB MIC for each strain.

PCR and sequencing. Primers were designed to target an $800 \mathrm{bp}$ region of the $e m b B$ gene encompassing codons $297-497$ for analysis. A pair of primers, embB-F (5'-GGT GAT ATT CGG CTT CCT- $\left.3^{\prime}\right)$ and embB-R (5'-ATA GCG CGG TGA TCA AAA AG-3'), was designed for the PCR. The $e m b B 306,-406$ and -497 mutations have been found to be a main cause of EMB resistance. This study was focused on $800 \mathrm{bp}$ of the hot spot region and excluded the possible mutations in the remaining region of the $3.3 \mathrm{~kb} \mathrm{embB}$ gene.

In a total volume of $20 \mu \mathrm{l}$, PCR mixtures contained $500 \mathrm{mM} \mathrm{KCl}$, $100 \mathrm{mM}$ Tris/ $\mathrm{HCl}$ (pH 9.0), $1 \%$ Triton X-100, $200 \mu \mathrm{M}$ deoxynucleoside triphosphates (dATP, dGTP, dTTP and dCTP), 10 pmol of each of the members of the primer pair, $1.5 \mathrm{mM} \mathrm{MgCl}_{2}, 1 \mathrm{U}$ Taq DNA polymerase (Qiagen) and $1 \mu \mathrm{l}$ template DNA. After heating the mixture to $95{ }^{\circ} \mathrm{C}$ for $7 \mathrm{~min}$, amplification was carried out in 35 sequential cycles with denaturation at $94{ }^{\circ} \mathrm{C}$ for $30 \mathrm{~s}$, primer annealing at $56{ }^{\circ} \mathrm{C}$ for $30 \mathrm{~s}$ and primer extension at $72{ }^{\circ} \mathrm{C}$ for $1 \mathrm{~min}$. The last extension step was performed by incubation at $72{ }^{\circ} \mathrm{C}$ for $7 \mathrm{~min}$. The DNA fragments were routinely examined on agarose gels stained with ethidium bromide. The direct sequencing of the $e m b B$ gene with PCR products was performed using a BigDye Terminator v3.1 Cycle Sequencing kit and analysed with an ABI 3730XL genetic analyser.

Statistical analysis. Statistical analyses were performed using SAS version 9.1.3. We performed the trend analysis using Bartholomew's test, which is a more powerful test than the chi-square test for ordered data, and also employed the Wilcoxon rank sum test to analyse MIC differences by the $e m b B$ mutation.

\section{RESULTS}

\section{Analysis of the embB mutation in EMB-susceptible $M$. tuberculosis strains}

A total of 124 EMB-susceptible and 93 EMB-resistant $M$. tuberculosis strains were analysed to determine a potential relationship between EMB resistance and $e m b B$ mutation. Two $(2.8 \%)$ of 72 pan-susceptible M. tuberculosis strains possessed $e m b B$ mutations at codons 360 (silent mutation, Val to Val) and 459 (Gly to Asp) (Table 1). These two strains revealed MICs of $1.25 \mu \mathrm{g} \mathrm{ml}^{-1}$ and $0.75 \mu \mathrm{g} \mathrm{ml}^{-1}$, which were not higher than the MICs for pan-susceptible M. tuberculosis strains without $e m b B$ mutation (Table 2). Two strains $(9.1 \%)$ out of 22 any-drug-resistant EMBsusceptible $M$. tuberculosis strains had embB mutations (Table 1). These strains were rifampicin mono-resistant with an embB406 mutation. MICs for these strains were higher than those for pan-susceptible strains (Table 2).

Nine $(30.0 \%)$ of 30 MDR but EMB-susceptible $M$. tuberculosis strains revealed various embB mutations such as Met306Ile, Met306Val, Tyr319Ser, Gly406Asp, Gly406Ala and Gln497Lys (Table 2). Only one strain (3.8\%) was EMBsusceptible out of 26 strains with Met306Ile mutation (Table 1). Two (6.3\%) of 32 strains with Met306Val mutation were MDR but EMB-susceptible. One (50\%) of two strains with Tyr319Ser mutation was EMB-susceptible and had a higher MIC $\left(1.5 \mu \mathrm{g} \mathrm{ml}^{-1}\right)$ than the MICs for pan-susceptible strains.

Four $(66.7 \%)$ of six Gly406Asp mutants (excluding embB405, 406 simultaneous mutant) and two (66.7\%) of three Gly406Ala mutants were EMB-susceptible. embB406 mutation seems to be less associated with EMB resistance at the LJ medium critical concentration $\left(2.0 \mu \mathrm{g} \mathrm{ml}^{-1}\right)$ in this study. One (50.0\%) of two strains with a Gln497Lys mutation was EMB-susceptible. However, all other Gln497Pro and Gln497Arg mutants revealed EMB resistance (Table 1).

\section{embB mutation rate according to drug resistance}

The embB mutation rate for 93 EMB-resistant M. tuberculosis strains was $90.3 \%$, but $2.8 \%$ in pan-susceptible strains, $9.1 \%$ in any-drug-resistant but non-MDR strains and $30.0 \%$ in MDR but EMB-susceptible M. tuberculosis strains (Table 1). The proportion of strains with $e m b B$ mutations increased with increasing anti-tuberculous drug resistance (i.e. in the order of pan-susceptible, any-drugresistant EMB-susceptible, MDR EMB-susceptible and EMB-resistant $M$. tuberculosis strains) as shown by Bartholomew's test (a statistical value of 141.65, higher than the critical value, which is in the range 3.805-4.354).

In the 93 EMB-resistant strains, the embB306 mutation was most prevalent ( $67.9 \%$ of strains), followed by the embB497 
Table 1. Distribution of the embB mutations

S, Susceptible; R, resistant; MDR, multidrug-resistant.

\begin{tabular}{|c|c|c|c|c|c|}
\hline \multirow[t]{2}{*}{ Amino acid change } & \multirow[t]{2}{*}{ Nucleotide change } & \multicolumn{3}{|c|}{ EMB-S } & \multirow[t]{2}{*}{ EMB-R (93) } \\
\hline & & Pan-S (72) & $\begin{array}{l}\text { Any-drug-R but } \\
\text { non-MDR (22) }\end{array}$ & MDR (30) & \\
\hline Met306Ile & ATG-ATC & & & & 6 \\
\hline Met306Ile & ATG-ATA & & & 1 & 18 \\
\hline Met306Ile & ATG-ATT & & & & 1 \\
\hline Met306Leu & ATG-CTG & & & & 2 \\
\hline Met306Val & ATG-GTG & & & 2 & 30 \\
\hline Tyr319Asp & TAT-GAT & & & & 1 \\
\hline Tyr319Ser & TAT-TCT & & & 1 & 1 \\
\hline Asp354Ala & GAC-GCC & & & & 3 \\
\hline Val360Val & GTG-GTA & $1^{*}$ & & & \\
\hline Asn399Thr $\dagger$ & AAC-ACC & & & & 2 \\
\hline Glu405Asp $\dagger$ & GAG-GAC & & & & 1 \\
\hline Glu405Asp, Gly406Asp & GAG-GAC, GGC-GAC & & & & 1 \\
\hline Gly406Asp & GGC-GAC & & 2 & 2 & 2 \\
\hline Gly406Ala & GGC-GCC & & & 2 & 1 \\
\hline Gly406Ser & GGC-AGC & & & & 2 \\
\hline Gly406Gly & GGC-GGA & & & & $1^{*}$ \\
\hline Gly459Asp $\dagger$ & GGC-GAC & 1 & & & \\
\hline Gln497Lys & CAG-AAG & & & 1 & 1 \\
\hline Gln497Pro & CAG-CCG & & & & 2 \\
\hline Gln497Arg & CAG-CGG & & & & 9 \\
\hline Mutation total & & $2(2.8 \%)$ & $2(9.1 \%)$ & $9(30.0 \%)$ & $84(90.3 \%)$ \\
\hline None & & $70(97.2 \%)$ & $20(90.9 \%)$ & $21(70.0 \%)$ & $9(9.7 \%)$ \\
\hline
\end{tabular}

*Synonymous mutation.

$\dagger$ Novel mutation.

mutation (14.3\%) and the embB406 mutation (7.1\%) (Table 3). The rate of $e m b B$ mutation in EMB-resistant strains also increased in proportion to the number of drugs that the strains were resistant to (a statistical value of 76.21, higher than the critical value of 3.82 by Bartholomew's test).

\section{Comparison of MICs}

The mean MICs for six pan-susceptible strains versus 11 EMB-susceptible strains but with other drug resistances and $e m b B$ mutations at codons $306,319,406$ or 497 were $1.0 \mu \mathrm{g}$ $\mathrm{ml}^{-1}$ and $1.42 \mu \mathrm{g} \mathrm{ml}^{-1}$, respectively. This result shows that the $e m b B$ mutation in the drug-resistant $M$. tuberculosis strains is associated with increased MICs of EMB (Wilcoxon statistic $=29.0, P$-value $=0.0052)$, even though the critical concentration for EMB-susceptible strains was $2.0 \mu \mathrm{g} \mathrm{ml}^{-1}$.

\section{Novel embB mutations and EMB susceptibility}

We identified novel embB mutations at codons 399, 405 and 459. We found two Asn399Thr and two Glu405Asp embB mutations in EMB-resistant $M$. tuberculosis strains. One Gly459Asp embB mutation was found in pan-susceptible $M$. tuberculosis strains.

\section{DISCUSSION}

The ever-increasing burden of drug resistance is a serious concern in the world, particularly for patients with $M$. tuberculosis infection. This mycobacterium uses various mechanisms to evade killing by therapeutic drugs, including mutations in genes that encode drug target proteins (Cole et al., 1998; Morris et al., 1995; Musser, 1995; Shi et al., 2007). The objective of this study was to identify mutations in a drug target gene in strains of $M$. tuberculosis prevalent among the Korean population. $e m b B$ gene mutation was believed to be the main cause of EMB resistance in $M$. tuberculosis. However, the relationship between embB gene mutation and EMB resistance is controversial because of embB mutation in EMB-susceptible M. tuberculosis strains (Mokrousov et al., 2002; Lee et al., 2004; Van Rie et al., 2001; Shi et al., 2007; Hazbón et al., 2005).

Hazbón et al. (2005) reported that 46 of $100 \mathrm{embB306}$ mutants were EMB-susceptible while 54 were EMB-resistant. After retesting, a few of the mutants were reclassified from susceptible to resistant. The EMB MICs for the embB306 mutants ranged from $<1$ to $>32 \mu \mathrm{g} \mathrm{ml}^{-1}$ (Hazbón et al., 2005). embB306 mutation was considered a common polymorphism with no relation to EMB resistance by Srivastava et al. (2009). 
Table 2. EMB MIC for M. tuberculosis strains retested in duplicate on $\sqcup$ medium after 5 weeks culture

\begin{tabular}{|c|c|c|c|}
\hline ID & $\begin{array}{l}\text { Phenotype } \\
\text { of DST }\end{array}$ & $\begin{array}{l}e m b B \text { mutation } \\
\text { codon and type }\end{array}$ & $\begin{array}{l}\text { Mean MIC of duplicate } \\
\text { retest }\left(\mu \mathrm{g} \mathrm{ml}^{-1}\right)\end{array}$ \\
\hline KMRC 00119-90955 & Pan-S & None & 1.25 \\
\hline KMRC 00119-90995 & Pan-S & None & 0.75 \\
\hline KMRC 00119-90807 & Pan-S & None & 1.0 \\
\hline KMRC 00119-90808 & Pan-S & None & 1.0 \\
\hline KMRC 00119-90999 & Pan-S & Val360Val (GTG-GTA) & 1.25 \\
\hline KMRC 00119-90806 & Pan-S & Gly459Asp (GGC-GAC) & 0.75 \\
\hline KMRC 00119-89635 & $\mathrm{R}$ & Gly406Asp (GGC-GAC) & 1.75 \\
\hline KMRC 00119-90870 & $\mathrm{R}$ & Gly406Asp (GGC-GAC) & 1.63 \\
\hline KMRC 00119-84132 & IR (MDR) & Met306Ile (ATG-ATA) & 1.5 \\
\hline KMRC 00119-87595 & IR (MDR) & Met306Val (ATG-GTG) & 1.5 \\
\hline KMRC 00119-89732 & IR (MDR) & Met306Val (ATG-GTG) & 0.88 \\
\hline KMRC 00119-88533 & IR (MDR) & Tyr319Ser (TAT-TCT) & 1.5 \\
\hline KMRC 00119-83962 & IR (MDR) & Gly406Asp (GGC-GAC) & 0.88 \\
\hline KMRC 00119-84671 & IR (MDR) & Gly406Asp (GGC-GAC) & 1.38 \\
\hline KMRC 00119-86964 & IR (MDR) & Gly406Ala (GGC-GCC) & 1.75 \\
\hline KMRC 00119-89589 & IR (MDR) & Gly406Ala (GGC-GCC) & 1.5 \\
\hline KMRC 00119-86862 & IRS (MDR) & Gln497Lys (CAG-AAG) & 1.38 \\
\hline KMRC 00119-84185 & IE & None & 8 \\
\hline KMRC 00119-89348 & IRE & Met306Ile (ATG-ATC) & 4 \\
\hline KMRC 00119-90333 & IRE & Met306Ile (ATG-ATA) & 6 \\
\hline KMRC 00119-87849 & ISE & Met306Leu (ATG-CTG) & 6 \\
\hline KMRC 00119-90084 & IRE & Met306Val (ATG-GTG) & 6 \\
\hline KMRC 00119-83615 & IRSE & Asn399Thr (AAC-ACC) & 12 \\
\hline KMRC 00119-88939 & IRSE & Asn399Thr (AAC-ACC) & 6 \\
\hline KMRC 00119-88791 & IE & Gly406Ala (GGC-GCC) & 4 \\
\hline KMRC 00119-84589 & IRE & Gln497Lys (CAG-AAG) & 6 \\
\hline KMRC 00119-89319 & IRSE & Gln497Pro (CAG-CGG) & 12 \\
\hline
\end{tabular}

${ }^{\star}$ DST, Drug-susceptibility testing; Pan-S, pan-susceptible; I, isoniazid-resistant; R, rifampicin-resistant; $\mathrm{S}$, streptomycin-resistant; E, ethambutol-resistant.

Depending upon mutation site or type, EMB resistance was found to be different. We found two embB mutations among 72 pan-susceptible M. tuberculosis strains. One of

Table 3. Distribution of mutation codons in 93 EMB-resistant strains

\begin{tabular}{|lcccc|}
\hline \multirow{2}{*}{$\begin{array}{l}\text { Mutation } \\
\text { codon }\end{array}$} & \multicolumn{3}{c}{ Drug resistance } & \multirow{2}{*}{ Total } \\
\cline { 2 - 4 } & EMB mono Non-MDR & MDR \\
\hline 306 & 2 & 23 & 32 & $57(67.9 \%)$ \\
319 & 1 & & 1 & $2(2.4 \%)$ \\
354 & & 1 & 2 & $3(3.6 \%)$ \\
399 & & & 2 & $2(2.4 \%)$ \\
405 & & & 1 & $1(1.2 \%)$ \\
405,406 & & & 1 & $1(1.2 \%)$ \\
406 & & 1 & 5 & $6(7.1 \%)$ \\
497 & & & 12 & $12(14.3 \%)$ \\
Total & $3(3.6 \%)$ & $25(29.8 \%)$ & $56(66.7 \%)$ & $84(100.0 \%)$ \\
Wild-type & & 4 & 5 & 9 \\
\end{tabular}

the two strains had a synonymous mutation at codon 360, which did not affect EMB resistance. The second pansusceptible strain had a Gly459Asp mutation, which is a novel mutation. However, this mutation also did not affect EMB resistance. $e m b B$ mutation in pan-susceptible strains in particular may not affect EMB resistance. Similar to our results, Hazbón et al. (2005) also could not find embB306 mutation in 582 pan-susceptible $M$. tuberculosis strains. Our findings suggest that certain mutations of the embB gene may not affect EMB resistance as had been postulated in previous reports (Hazbón et al., 2005; Sekiguchi et al., 2007).

Only three MDR isolates (5.0\%) out of $60 \mathrm{embB306}$ mutants were EMB-susceptible in this study. Even though we also detected the embB306 mutation in EMB-susceptible clinical isolates, an embB306 substitution experiment from wildtype to mutation type proved that embB306 mutation increased the EMB MIC (Safi et al., 2008; Starks et al., 2009). Safi et al. (2008) found that the substitution at embB306 did not affect rifampicin or isoniazid MICs. Further, we found that $\operatorname{six}(54.5 \%)$ out of $11 \mathrm{embB} 406$ mutant strains (excluding two strains, one with a synonymous mutation 
and another with an additional mutation at embB405) were EMB-susceptible (Table 1). embB406 mutations have also been detected previously in EMB-susceptible isolates (Lee et al., 2004; Shi et al., 2011; Ramaswamy et al., 2004). We found only one EMB-susceptible $(7.7 \%)$ strain out of 13 embB497 mutants. In a recent study, embB406 and allelic embB497 exchange increased the EMB MIC 3-3.5-fold and 6-fold, respectively, over that for the wild-type $e m b B$ gene and these allelic exchanges did not affect the MIC of other anti-tuberculous drugs such as isoniazid or rifampicin (Safi et al., 2010).

For the minor mutations, two of three embB319 mutants were EMB-resistant and another one was EMB-susceptible with MDR as shown in Table 2. Likewise, the Tyr319Ser and Tyr319Asp mutations were found in EMB-resistant $M$. tuberculosis strains in previous reports (Plinke et al., 2006; Sugawara et al., 2005). The Asp354Ala mutation revealed EMB resistance, which has been reported previously (Sekiguchi et al., 2007).

In this study, three novel embB mutations (Asn399Thr, Glu405Asp and Gly459Asp) were detected. Though all three of the codon sites were also found in another study, those mutations produced different amino acids (Srivastava et al., 2009). Among the three novel mutations, two (Asn399Thr and Glu405Asp) were associated with EMB resistance.

Our study further suggested that the proportion of strains with $e m b B$ mutations increased with the increasing number of drug resistances in both EMB-susceptible and EMBresistant strains. This finding also agreed with those in other studies (Lee et al., 2004; Shi et al., 2011; Shen et al., 2007).

Along with the above results, we found that the mean EMB MIC for embB-mutated EMB-susceptible strains was higher than that for pan-susceptible strains. This may be caused by the limitation of conventional drug susceptibility testing methods for EMB (Kim, 2005). Kim (2005) reported that $90 \%$ of probably EMB-susceptible strains and $30-50 \%$ of probably EMB-resistant strains were inhibited at the critical concentration of $2.0 \mu \mathrm{g} \mathrm{ml}^{-1}$ on LJ solid medium.

However, we compared only the MICs for EMB-susceptible strains with those for pan-susceptible and other-drugresistant strains with the $e m b B$ mutation, so we need further studies to compare the MICs of EMB in EMB-susceptible strains with and without the $e m b B$ mutations.

In conclusion, the exact mechanism of EMB resistance is not yet clearly understood. Our data support that the embB mutation, except for a few specific mutations such as Gly459Asp, may be the main cause of EMB resistance.

\section{ACKNOWLEDGEMENTS}

This work was funded by the Korean Government (MEST), Korea Research Foundation (KRF) Grant No. 2009-0075492.

\section{REFERENCES}

Alcaide, F., Pfyffer, G. E. \& Telenti, A. (1997). Role of $e m b B$ in natural and acquired resistance to ethambutol in mycobacteria. Antimicrob Agents Chemother 41, 2270-2273.

Canetti, G., Fox, W., Khomenko, A., Mahler, H. T., Menon, N. K., Mitchison, D. A., Rist, N. \& Smelev, N. A. (1969). Advances in techniques of testing mycobacterial drug sensitivity, and the use of sensitivity tests in tuberculosis control programmes. Bull World Health Organ 41, 21-43.

CDC (2006). Emergence of Mycobacterium tuberculosis with extensive resistance to second-line drugs - worldwide, 2000-2004. MMWR Morb Mortal Wkly Rep 55, 301-305.

Cole, S. T., Brosch, R., Parkhill, J., Garnier, T., Churcher, C., Harris, D., Gordon, S. V., Eiglmeier, K., Gas, S. \& other authors (1998). Deciphering the biology of Mycobacterium tuberculosis from the complete genome sequence. Nature 393, 537-544.

Hazbón, M. H., Bobadilla del Valle, M., Guerrero, M. I., Varma-Basil, M., Filliol, I., Cavatore, M., Colangeli, R., Safi, H., Billman-Jacobe, H. \& other authors (2005). Role of embB codon 306 mutations in Mycobacterium tuberculosis revisited: a novel association with broad drug resistance and IS6110 clustering rather than ethambutol resistance. Antimicrob Agents Chemother 49, 3794-3802.

Kemper, C. A., Havlir, D., Haghighat, D., Dubé, M., Bartok, A. E., Sison, J. P., Yao, Y., Yangco, B., Leedom, J. M. \& other authors (1994). The individual microbiologic effect of three antimycobacterial agents, clofazimine, ethambutol, and rifampin, on Mycobacterium avium complex bacteremia in patients with AIDS. J Infect Dis 170, 157-164.

Kim, S. J. (2005). Drug-susceptibility testing in tuberculosis: methods and reliability of results. Eur Respir J 25, 564-569.

Lee, A. S., Othman, S. N., Ho, Y. M. \& Wong, S. Y. (2004). Novel mutations within the $e m b B$ gene in ethambutol-susceptible clinical isolates of Mycobacterium tuberculosis. Antimicrob Agents Chemother 48, 4447-4449.

Migliori, G. B., Loddenkemper, R., Blasi, F. \& Raviglione, M. C. (2007). 125 years after Robert Koch's discovery of the tubercle bacillus: the new XDR-TB threat. Is "science" enough to tackle the epidemic? Eur Respir J 29, 423-427.

Mokrousov, I., Otten, T., Vyshnevskiy, B. \& Narvskaya, O. (2002). Detection of embB306 mutations in ethambutol-susceptible clinical isolates of Mycobacterium tuberculosis from Northwestern Russia: implications for genotypic resistance testing. J Clin Microbiol 40, 3810-3813.

Morris, S., Bai, G. H., Suffys, P., Portillo-Gomez, L., Fairchok, M. \& Rouse, D. (1995). Molecular mechanisms of multiple drug resistance in clinical isolates of Mycobacterium tuberculosis. J Infect Dis 171, 954960 .

Musser, J. M. (1995). Antimicrobial agent resistance in mycobacteria: molecular genetic insights. Clin Microbiol Rev 8, 496-514.

Park, Y. K., Bai, G. H. \& Kim, S. J. (2000). Restriction fragment length polymorphism analysis of Mycobacterium tuberculosis isolated from countries in the western pacific region. J Clin Microbiol 38, 191-197.

Plinke, C., Rüsch-Gerdes, S. \& Niemann, S. (2006). Significance of mutations in $e m b B$ codon 306 for prediction of ethambutol resistance in clinical Mycobacterium tuberculosis isolates. Antimicrob Agents Chemother 50, 1900-1902.

Rabarijaona, L., Boisier, P., Ratsirahonana, O., Razafinimanana, J., Rakotomanana, F., Ratsitorahina, M., Ramarokoto, H., Cauchoix, B. \& Aurégan, G. (1999). Replacement of streptomycin by ethambutol in the intensive phase of tuberculosis treatment: no effect on compliance. Int J Tuberc Lung Dis 3, 42-46. 
Ramaswamy, S. V. \& Musser, J. M. (1998). Molecular genetic basis of antimicrobial agent resistance in Mycobacterium tuberculosis: 1998 update. Tuber Lung Dis 79, 3-29.

Ramaswamy, S. V., Dou, S. J., Rendon, A., Yang, Z., Cave, M. D. \& Graviss, E. A. (2004). Genotypic analysis of multidrug-resistant Mycobacterium tuberculosis isolates from Monterrey, Mexico. J Med Microbiol 53, 107-113.

Raviglione, M. C. \& Smith, I. M. (2007). XDR tuberculosis implications for global public health. $N$ Engl J Med 356, 656659.

Safi, H., Sayers, B., Hazbón, M. H. \& Alland, D. (2008). Transfer of embB codon 306 mutations into clinical Mycobacterium tuberculosis strains alters susceptibility to ethambutol, isoniazid, and rifampin. Antimicrob Agents Chemother 52, 2027-2034.

Safi, H., Fleischmann, R. D., Peterson, S. N., Jones, M. B., Jarrahi, B. \& Alland, D. (2010). Allelic exchange and mutant selection demonstrate that common clinical embCAB gene mutations only modestly increase resistance to ethambutol in Mycobacterium tuberculosis. Antimicrob Agents Chemother 54, 103-108.

Sekiguchi, J., Miyoshi-Akiyama, T., Augustynowicz-Kopeć, E., Zwolska, Z., Kirikae, F., Toyota, E., Kobayashi, I., Morita, K., Kudo, K. \& other authors (2007). Detection of multidrug resistance in Mycobacterium tuberculosis. J Clin Microbiol 45, 179-192.

Shen, X., Shen, G. M., Wu, J., Gui, X. H., Li, X., Mei, J., DeRiemer, K. \& Gao, Q. (2007). Association between $e m b B$ codon 306 mutations and drug resistance in Mycobacterium tuberculosis. Antimicrob Agents Chemother 51, 2618-2620.

Shi, R., Zhang, J., Otomo, K., Zhang, G. \& Sugawara, I. (2007). Lack of correlation between $e m b B$ mutation and ethambutol MIC in Mycobacterium tuberculosis clinical isolates from China. Antimicrob Agents Chemother 51, 4515-4517.

Shi, D., Li, L., Zhao, Y., Jia, Q., Li, H., Coulter, C., Jin, Q. \& Zhu, G. (2011). Characteristics of $e m b B$ mutations in multidrug-resistant
Mycobacterium tuberculosis isolates in Henan, China. I Antimicrob Chemother 66, 2240-2247.

Sreevatsan, S., Stockbauer, K. E., Pan, X., Kreiswirth, B. N., Moghazeh, S. L., Jacobs, W. R., Jr, Telenti, A. \& Musser, J. M. (1997). Ethambutol resistance in Mycobacterium tuberculosis: critical role of embB mutations. Antimicrob Agents Chemother 41, 1677-1681.

Srivastava, S., Ayyagari, A., Dhole, T. N., Nyati, K. K. \& Dwivedi, S. K. (2009). emb nucleotide polymorphisms and the role of embB306 mutations in Mycobacterium tuberculosis resistance to ethambutol. Int J Med Microbiol 299, 269-280.

Starks, A. M., Gumusboga, A., Plikaytis, B. B., Shinnick, T. M. \& Posey, J. E. (2009). Mutations at embB codon 306 are an important molecular indicator of ethambutol resistance in Mycobacterium tuberculosis. Antimicrob Agents Chemother 53, 1061-1066.

Sugawara, I., Otomo, K., Yamada, H., Wang, G., Du, C., Shi, R. \& Zhang, G. (2005). The molecular epidemiology of ethambutolresistant Mycobacterium tuberculosis in Henan Province, China. Jpn J Infect Dis 58, 393-395.

Telenti, A., Philipp, W. J., Sreevatsan, S., Bernasconi, C., Stockbauer, K. E., Wieles, B., Musser, J. M. \& Jacobs, W. R., Jr (1997). The emb operon, a gene cluster of Mycobacterium tuberculosis involved in resistance to ethambutol. Nat Med 3, 567-570.

Van Rie, A., Warren, R., Mshanga, I., Jordaan, A. M., van der Spuy, G. D., Richardson, M., Simpson, J., Gie, R. P., Enarson, D. A. \& other authors (2001). Analysis for a limited number of gene codons can predict drug resistance of Mycobacterium tuberculosis in a highincidence community. J Clin Microbiol 39, 636-641.

WHO (1997). Treatment of Tuberculosis. Guidelines for the Management of Drug-resistant Tuberculosis, 2nd edn, pp. 1-47. Geneva: World Health Organization.

WHO (2009). Global Tuberculosis Control 2009: Epidemiology Strategy Financing. Report No. WHO/HTM/TB/2009.411, pp. 1-303. Geneva: World Health Organization. 